Ciência Florestal, Santa Maria, v. 24, n. 1, p. 171-183, jan.-mar., 2014

ISSN 0103-9954

\title{
UTILIZAÇÃO DE GEOPROCESSAMENTO NA SIMULAÇÃO DE ÁREAS DE CLAREIRAS EM UM SISTEMA DE MANEJO EM REGIME JARDINADO
}

\author{
GEOPROCESSING USE FOR THE SIMULATION OF GAPS AREAS IN A MANAGEMENT \\ GARDENING SYSTEM
}

Henrique Luis Godinho Cassol ${ }^{1}$ Frederico Dimas Fleig ${ }^{2}$

\begin{abstract}
RESUMO
O objetivo deste trabalho foi simular as áreas de clareiras formadas, pós-exploração, em um Sistema de Manejo em Regime Jardinado, utilizando técnicas de Geoprocessamento. Em 5,6 ha de uma Floresta Estacional Semidecidual, localizada na Linha Canudos, Santa Maria/RS, foram georreferenciadas 924 árvores com o uso de um receptor GPS. Destas, determinou-se a taxa de corte sustentado pela distribuição das frequências balanceadas por classe diamétrica e foram selecionadas 82 árvores para exploração. Nas árvores selecionadas para o corte, as áreas de clareiras formadas pela liberação do dossel e as áreas de clareiras decorrentes do impacto, ocasionado pela queda, foram simuladas em um ambiente SIG, empregando-se como técnica de exploração de impacto reduzido, o abate dirigido das árvores. Ao todo, 98 indivíduos $\left(32 \mathrm{~m}^{3}\right)$ serão beneficiados pela liberação do dossel e 63 indivíduos $\left(12,07 \mathrm{~m}^{3}\right)$ serão impactados pela queda das árvores exploradas. Portanto, conclui-se que o uso de técnicas de Geoprocessamento aplicadas ao manejo de florestas nativas é um importante recurso quando se deseja intervir na floresta, com o objetivo de reduzir os impactos decorrentes da exploração.
\end{abstract}

Palavras-chave: manejo florestal sustentado; SIG; exploração de impacto reduzido; clareiras.

\begin{abstract}
The aim of this study was to simulate the gap areas formed after logging in a management gardening system, using geoprocessing techniques. In a 5.6 ha of a seasonal semi deciduous forest located in Linha Canudos, Santa Maria, Rio Grande do Sul state, 924 trees were georeferenced using a GPS receiver. In these trees was determined the sustainable cutting rate beyond balanced distribution frequencies by diameter class selecting 82 cutting trees through this analysis. At the selected cutting trees, the releasing areas of canopy gaps and the impacting areas were estimated from falling cutting trees using GIS and reduced impact logging techniques as known as directed tree felling. Altogether, 98 trees $\left(32 \mathrm{~m}^{3}\right)$ will benefit from releasing of canopy and 63 trees $\left(12.07 \mathrm{~m}^{3}\right)$ will be impacted by the falling of the explored trees. Therefore, it is concluded that the geoprocessing techniques applied to native forest management is an important feature when you want to intervene in the forest, aiming to reduce the forest cutting impact.
\end{abstract}

Keywords: sustainable forest management; GIS; reduced impact logging; tree falling gaps; canopy gaps.

1 Engenheiro Florestal, Mestrando do Programa de Pós-Graduação em Sensoriamento Remoto, Universidade Federal do Rio Grande do Sul, Av. Bento Gonçalves, 9500, CEP 91501-970, Porto Alegre (RS), Brasil. hlcassol@hotmail.com

2 Engenheiro Florestal, Dr., Professor Adjunto do Departamento de Ciências Florestais, Centro de Ciências Rurais, Universidade Federal de Santa Maria, Av. Roraima, 1000, CEP 97105-900, Santa Maria (RS), Brasil. dimasfleig@uol.com.br

Recebido para publicação em 27/08/2010 e aceito em 26/03/2013 


\section{INTRODUÇÃO}

Sistema de Manejo em Regime Jardinado (SMRJ) é um sistema de manejo para florestas heterogêneas e inequiâneas, com intervenções baseadas no corte seletivo, regeneração natural ou artificial, visando à produção contínua de produtos florestais e à manutenção da biodiversidade de espécies. É o único sistema de manejo legal para exploração de florestas naturais do Rio Grande do Sul (RIO GRANDE DO SUL, 2012).

Neste tipo de manejo, a regeneração natural ocorre de maneira espontânea e sustentável (autorrenovação) e as árvores possuem crescimento lento na juventude e acelerado após a liberação do dossel (SCHÜTZ, 1989). Schneider e Finger (2000) destacam que um SMRJ só é possível quando se conhecem os crescimentos periódicos anuais e médios anuais de todas as espécies pertencentes à unidade de manejo. Assim, a taxa de corte sustentado pode ser estabelecida com base nas frequências balanceadas por classes de diâmetro das espécies e a função de Meyer (1958) fornece o cálculo base para a determinação desta taxa.

O uso do Geoprocessamento, conciliado com o uso do sistema de posicionamento por satélite (GPS) e de um ambiente de Sistema de Informações Geográficas (SIG), é uma importante ferramenta ao se aplicar em um SMRJ, no que tange à precisão do mapeamento e à localização das árvores da floresta, assim como na maior capacidade visual para a tomada de decisões sobre as intervenções silviculturais e de exploração, que tenham por objetivo a sustentabilidade da produção e a diminuição dos impactos pós-exploração.

Um exemplo da integração de planejamento do manejo florestal de forma digital com a utilização de um SIG foi desenvolvido pela EMBRAPA/ Acre em parceria com outras instituições e foi denominado de Modelo Digital de Exploração Florestal - MODEFLORA (FIGUEIREDO et al., 2008).

O manejo em florestas tropicais tem avançado muito quanto às pesquisas básicas relacionadas à avaliação da regeneração e à dinâmica da floresta pós-exploração, no sentido de reduzir os impactos da exploração sobre a floresta remanescente (OLIVEIRA e BRAZ, 1998). Isto é comprovado por Jardim et al. (1993), os quais destacam que a atividade de exploração florestal influencia diretamente na diversidade de espécies, decorrente também da dimensão das clareiras (WHITMORE,
1978, 1989; DENSLOW, 1980) e do tipo de exploração, se convencional ou planejada (VIDAL et al., 1998).

A abertura das clareiras e os danos residuais provenientes da exploração florestal são minimizados quando estes danos se concentram em uma mesma área, por meio do abate dirigido das árvores (JACKSON et al., 2002). Pereira et al. (2002) citam que o abate dirigido das árvores é uma técnica de exploração de impacto reduzido (EIR) com a finalidade de reduzir os danos aos indivíduos remanescentes, pois a queda da árvore é dirigida para áreas abertas ou clareiras já formadas.

A EIR consiste em uma série de diretrizes pré e pós-exploratórias orientadas ao planejamento das operações, ao treinamento de recursos humanos e ao investimento no Manejo Florestal. A EIR tem por objetivo: minimizar os danos ambientais, conservar o potencial de exploração futura, manter os serviços da floresta, reduzir os custos operacionais da exploração, aumentar a eficácia do trabalho e reduzir o desperdício (SABOGAL et al., 2000). Estes mesmos autores demonstraram que esta técnica não é necessariamente mais cara do que a de exploração convencional.

Segundo Bulfe et al. (2009), a taxa de mortalidade dos indivíduos remanescentes é influenciada por uma maior mortalidade das árvores que sofreram danos severos e está diretamente relacionada à dimensão da clareira formada. Assim, as áreas com uma intensidade de exploração baixa e controlada possuem taxas de mortalidade similares às observadas nas áreas-testemunha sem intervenção, comprovando-se que a redução nos danos diminui a mortalidade pós-exploração (BERTAULT e SIST, 1997; SIST et al., 1998; SIST e NGUYEN-THÉ, 2002).

Portanto, o método para estimar a dimensão da clareira tem sido um importante foco de atenção a engenheiros florestais e ecologistas, porque a precisão nas estimativas das dimensões das clareiras pós-exploração é a base para a taxa de regeneração da floresta (VEBLEN, 1989; VALAVERDE e SILVERTOWN, 1997; LIMA, 2005; HU e ZHU, 2008). Alguns autores estabeleceram fortes relações morfométricas e interdimensionais para estimar o diâmetro e a área de copa em árvores nativas da floresta ombrófila mista (LONGHI, 1980) e estacional semidecidual (DURLO, 1996; DURLO et al., 2009) e, dessa forma, determinar a dimensão da clareira formada, a partir da área projetada de copa.

Pesquisas recentes consistem no registro das dimensões das clareiras mediante uso de méto- 
dos elipsoidais (RUNKLE, 1981, 1992), a partir de fotografias hemisféricas (ZHU et al., 2009; BULFE, 2009), ou a partir do registro de imagens obtidas de sensores remotos (BETTS et al., 2005). Betts et al. (2005) detectaram as clareiras formadas, pós-exploração, de forma rápida e acurada, por meio de variações texturais nos pixels de uma fotografia de alta resolução espacial, com base em uma classificação supervisionada por redes neurais e concluíram que a precisão do método está diretamente relacionada à resolução espacial da imagem e à dimensão do filtro textural empregado.

As dimensões das clareiras formadas, pós-exploração, são correlacionadas significativa e positivamente com a densidade e a altura do dossel e que, de qualquer forma, a curva de distribuição das clareiras em classes de tamanho segue um padrão exponencial decrescente, com maior concentração nas menores classes de tamanho (MARTINS, 1999).

Sendo assim, neste trabalho foram utilizadas ferramentas que compreendem o universo de um SIG para simular, quantificar e espacializar, geograficamente, as áreas de clareiras formadas pelas árvores exploráveis e avaliar o possível impacto que as mesmas ocasionariam nas árvores remanescentes.

\section{MATERIAIS E MÉTODOS}

O trabalho foi realizado em 5,65 ha de uma propriedade localizada na Linha Canudos, distrito do município de Santa Maria/RS, próximo ao município de Itaara. Está localizada em uma área de transição entre a Depressão Periférica e o Planalto Meridional, a Escarpa do Planalto, tendo como característica a acentuada declividade. A área de estudo localiza-se entre as coordenadas geográficas

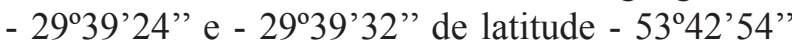
e - 53'42'44" de latitude.

O censo consistiu na colocação de plaquetas numeradas em todas as árvores que contivessem diâmetros a altura do peito (DAP) superiores a $15 \mathrm{~cm}$. Além disso, foram coletados dados das alturas comerciais (Hc), alturas totais (Ht), qualidade de fuste (QF), número do ponto do GPS e direção da árvore em relação à picada virtual.

As picadas foram criadas virtualmente na tela do GPS, facilitando a localização das árvores a campo e reduzindo o tempo do inventário. Os números dos pontos do GPS, correspondentes às plaquetas numeradas e às árvores inventariadas, foram tomados utilizando um receptor GPS Garmim modelo 76Csx e transformados em arquivo shapefile.
Este modelo de GPS é dotado de uma antena de alta sensibilidade, equipada com um microchip que decodifica sinais GPS de baixa frequência (menores de $160 \mathrm{dBm}$ ), possibilitando a penetração do sinal no interior da floresta. Possui também uma tecnologia denominada de protocolo Sirf Star III, que recalcula automaticamente a posição do receptor, melhorando significativamente o sinal e a precisão do aparelho inferior a $5 \mathrm{~m}$ (FIGUEIREDO et al., 2008).

$\mathrm{O}$ inventário foi realizado tendo como base o cumprimento dos dispositivos legais dos planos de manejo no Rio Grande do Sul, através do Capítulo II de Exploração e Reposição Florestal do Código Florestal Estadual de 21 de Janeiro de 1992, Lei 9.519, quanto ao artigo que segue (RIO GRANDE DO SUL, 1992):

Art. $7^{\circ}$ - A autorização para a exploração das florestas nativas somente será concedida através de sistema de manejo em regime jardinado, não sendo permitido o corte raso, havendo a obrigatoriedade de reposição nos termos desta Lei.

Na Tabela 1 são observados os parâmetros fitossociológicos da floresta, provenientes do inventário, que serviram como base para o SMRJ.

As árvores inventariadas foram categorizadas em cinco classes, conforme o preconizado pelo MODEFLORA (FIGUEIREDO et al., 2008) e pela Instrução Normativa $n^{\circ} 5$, de 11 de dezembro de 2006, para aprovação de Planos de Manejo Florestais Sustentáveis, que foram:

1. Exploráveis (árvores com DAP superior a um valor pré-estabelecido, variável por espécie);

2. Remanescentes;

3. Porta-sementes $(10 \%$ dos indivíduos por espécie);

4. APP (pertencentes às áreas de preservação permanente - APPs, imunes ao corte);

5. Imunes (pertencentes à lista de espécies imunes ao corte do Rio Grande do Sul)

$\mathrm{O}$ volume comercial com casca por hectare (Vc) foi estimado com base nos DAPs e nas alturas comerciais $(\mathrm{Hc})$ das árvores, pela fórmula descrita por Schneider e Finger (2000) para espécies de folhosas da Floresta Nacional de Passo Fundo (Equação 1):

$\log V c:-3,95275+2,04354 \times \log (D A P)+0,61461 \times \log (H c)$

Os volumes comerciais com casca por hectare das árvores exploráveis, separados por classes de diâmetro e espécies, estão listados na Tabela 2. 
TABELA 1: Densidade, dominância e índice de valor de cobertura.

TABLE 1: Density, dominance and coverage value index.

\begin{tabular}{|c|c|c|c|c|c|c|}
\hline \multirow{2}{*}{ Cód. } & \multirow{2}{*}{ Espécie } & \multicolumn{2}{|c|}{ Densidade } & \multicolumn{3}{|c|}{ Dominância } \\
\hline & & Abs. (N) & Rel. $(\%)$ & Abs. $\left(\mathrm{m}^{2}\right)$ & Rel. (\%) & IVC $(\%)$ \\
\hline 1 & Enterolobium contortisiliquum (Vell.) Morong. & 111 & 26,52 & 754,69 & 30,90 & 28,71 \\
\hline 2 & Ocotea puberula(Rich.) Nees & 87 & 20,67 & 386,07 & 15,81 & 18,24 \\
\hline 3 & Desconhecida & 30 & 7,03 & 108,76 & 4,45 & 5,74 \\
\hline 4 & Phytolacca dioica $\mathrm{L}$. & 11 & 2,60 & 175,52 & 7,19 & 4,89 \\
\hline 5 & Nectandra megapotamica (Spreng.) Mez & 10 & 2,49 & 83,33 & 3,41 & 2,95 \\
\hline 6 & Cupania vernalis Cambess. & 12 & 2,92 & 64,83 & 2,65 & 2,79 \\
\hline 7 & Hovenia dulcis Thunb. & 10 & 2 & 75,00 & 3 & 2,78 \\
\hline 8 & Nectandra lanceolada Nees \& Mart. Ex Nees & 8 & 1,84 & 69,88 & 2,86 & 2,35 \\
\hline 9 & Matayba eleagnoides Radlk. & 12 & 2,92 & 43,27 & 1,77 & 2,35 \\
\hline 10 & Parapiptadenia rigida (Benth.) Brenan & 5 & 1,30 & 81,16 & 3,32 & 2,31 \\
\hline 11 & Morta & 9 & 2,16 & 57,88 & 2,37 & 2,27 \\
\hline 12 & Jacaranda micrantha Cham. & 12 & 2,81 & 33,57 & 1,37 & 2,09 \\
\hline 13 & Casearia sylvestris $\mathrm{Sw}$. & 11 & 2,60 & 33,55 & 1,37 & 1,99 \\
\hline 14 & Cabralea canjerana (Vell.) Mart. & 6 & 1,41 & 61,43 & 2,52 & 1,96 \\
\hline 15 & Campomanesia xanthocarpa (Mart.) Berg. & 7 & 1,73 & 46,80 & 1,92 & 1,82 \\
\hline 16 & Myrocarpus frondosus Allemao & 10 & 2,38 & 25,63 & 1,05 & 1,72 \\
\hline 17 & Cedrela fissilis Vell. & 6 & 1,41 & 38,63 & 1,58 & 1,49 \\
\hline 18 & Erythrina falcata Benth. & 3 & 0,76 & 50,10 & 2,05 & 1,40 \\
\hline 19 & Machaerium stipitatum (DC) Vogel & 7 & 1,62 & 21,78 & 0,89 & 1,26 \\
\hline 20 & Cordia americana $\mathrm{L}$. & 5 & 1,19 & 30,34 & 1,24 & 1,22 \\
\hline 21 & Ficus sp. & 4 & 0,87 & 37,63 & 1,54 & 1,20 \\
\hline 22 & Annona cf. salicifolia Schtdl. & 6 & 1,41 & 17,13 & 0,70 & 1,05 \\
\hline 23 & Psidium guajava $\mathrm{L}$. & 5 & 1,08 & 14,66 & 0,60 & 0,84 \\
\hline 24 & Cordia trichotmoma (Vell.) Arráb. ex steud. & 4 & 0,97 & 13,62 & 0,56 & 0,77 \\
\hline 25 & Luehea divaricata Mart. & 3 & 0,65 & 17,24 & 0,71 & 0,68 \\
\hline 26 & Zanthoxylum rhoifolium Lam. & 4 & 0,87 & 9,24 & 0,38 & 0,62 \\
\hline 27 & Allophylus edulis (A. St.-Hill., Camb. \& A. Juss.) Radlk. & 4 & 0,87 & 9,20 & 0,38 & 0,62 \\
\hline 28 & Eribotrya japonica Lindl. & 3 & 0,65 & 12,97 & 0,53 & 0,59 \\
\hline 29 & Eugenia uniflora L. & 2 & 0,43 & 13,34 & 0,55 & 0,49 \\
\hline 30 & Aiouea saligna Meisn. & 1 & 0,32 & 7,85 & 0,32 & 0,32 \\
\hline 31 & Campomanesia guazumifolia (Camb.) Legr. & 2 & 0,43 & 3,49 & 0,14 & 0,29 \\
\hline 32 & Diospyros brasiliensis Mart. & 0 & 0,11 & 10,59 & 0,43 & 0,27 \\
\hline 33 & Apuleia leiocarpa (Vogel) J. F. Macbr. & 1 & 0,32 & 3,47 & 0,14 & 0,23 \\
\hline 34 & Syagrus romanzoffiana (Cham.) Glassman & 1 & 0,22 & 3,41 & 0,14 & 0,18 \\
\hline 35 & Citrus aurantium $\mathrm{L}$. & 1 & 0,22 & 3,29 & 0,13 & 0,18 \\
\hline 36 & Sebastiania commersoniana (Baill.) L. B. Smith \& Downs & 1 & 0,22 & 1,28 & 0,05 & 0,13 \\
\hline 37 & Morus nigra $\mathrm{L}$. & 0 & 0,11 & 2,08 & 0,09 & 0,10 \\
\hline 38 & Boehmeria caudata $\mathrm{Sw}$. & 0 & 0,11 & 1,15 & 0,05 & 0,08 \\
\hline 39 & Eugenia involucrata DC. & 0 & 0,11 & 1,10 & 0,05 & 0,08 \\
\hline 40 & Citrus deliciosa Tenore & 0 & 0,11 & 0,92 & 0,04 & 0,07 \\
\hline 41 & Helietta apiculata Benth. & 0 & 0,11 & 0,92 & 0,04 & 0,07 \\
\hline & Total & 420 & 100 & 2443 & 100 & 100 \\
\hline
\end{tabular}

Em que: Abs. $=$ absoluta; Rel. $=$ relativa IVC $=$ índice de valor de cobertura. 
Com base na Tabela 2, as árvores representadas pela categoria das exploráveis foram utilizadas para a definição da taxa de corte sustentado do SMRJ, mediante a função de Meyer e as distribuições de volume por classe de diâmetro. O SMRJ consistiu na seleção de árvores para o abate (exploráveis) e na definição de alguns parâmetros ecológicos, como: o ciclo de corte, o diâmetro máximo de corte, por espécie, a área basal remanescente e o quociente Liocourt (q), de forma a garantir a produção sustentada de madeira.

O quociente de Liocourt expressa o melhor ajuste das distribuições das frequências de classes de diâmetro, em que o número de árvores por classe de diâmetro decresce seguindo uma progressão geométrica (SCHNEIDER e FINGER, 2000). Assim, um quociente de Liocourt de 2 significa que 50\% das árvores da classe de diâmetro precedente podem ser retiradas, mantendo-se a produção sustentada de madeira.
Por meio da função de Meyer, definiu-se um ciclo de corte de 25 anos, diâmetro máximo de corte de $60 \mathrm{~cm}$, área basal remanescente de $5 \mathrm{~m}^{2} /$ ha e um quociente de Liocourt calculado de 1,4. A taxa de corte ajustada para a função de Meyer foi de $12,51 \mathrm{~m}^{3} / \mathrm{ha}$ (Tabela 3 ).

Os indivíduos foram deliberadamente selecionados para o corte, conforme os volumes balanceados, por classe de diâmetro, oriundos da Tabela 4. Além disso, foram selecionados indivíduos de menor interesse comercial, preferivelmente, de má qualidade de fuste, objetivando beneficiar espécimes de maior relevância comercial para o segundo ciclo e aquelas espécies que contivessem maior índice de valor de cobertura (IVC), de forma a garantir a diversidade da floresta.

Os Planos de Informação (PI), tais como açudes, rios, estradas, limites, áreas de preservação permanente (APPs) e benfeitorias foram elaborados com o uso do GPS e pela transformação des-

TABELA 2: Volume por classe de diâmetro e espécie, por hectare.

TABLE 2: Volume by diameter class and species per hectare.

\begin{tabular}{|c|c|c|c|c|c|c|c|}
\hline \multirow{2}{*}{ Espécie } & \multicolumn{6}{|c|}{ Centro de Classe de DAP $(\mathrm{cm})$} & \multirow{2}{*}{ Total $\left(\mathrm{m}^{3} / \mathrm{ha}\right)$} \\
\hline & 20 & 30 & 40 & 50 & 60 & 70 & \\
\hline Enterolobium contortisiliquum (Vell.) Morong. & & 1,255 & 7,564 & 4,256 & 2,615 & - & 15,690 \\
\hline Ocotea puberula(Rich.) Nees & 1,421 & 2,846 & 0,081 & - & - & - & 4,348 \\
\hline Parapiptadenia rigida (Benth.) Brenan & 0,112 & - & 0,200 & 0,670 & 1,327 & 0,538 & 2,847 \\
\hline Nectandra megapotamica (Spreng.) Mez & 0,109 & 0,246 & 0,962 & 0,916 & - & - & 2,233 \\
\hline Desconhecida & 0,639 & 1,038 & 0,351 & - & - & - & 2,028 \\
\hline Nectandra lanceolada Nees \& Mart. Ex Nees & - & - & 0,406 & - & 1,301 & - & 1,707 \\
\hline Matayba eleagnoides Radlk. & 0,127 & 0,258 & 0,492 & - & - & - & 0,877 \\
\hline Cedrela fissilis Vell. & - & 0,132 & - & 0,484 & - & - & 0,616 \\
\hline Cupania vernalis Cambess. & 0,033 & 0,288 & 0,246 & - & - & - & 0,567 \\
\hline Aiouea saligna Meisn. & - & 0,367 & - & - & - & - & 0,367 \\
\hline Campomanesia xanthocarpa (Mart.) Berg. & - & 0,195 & 0,167 & - & - & - & 0,362 \\
\hline Machaerium stipitatum (DC) Vogel & 0,050 & 0,287 & - & - & - & - & 0,338 \\
\hline Albizia niopoides (Spruce ex Benth.) Burkart & 0,100 & 0,197 & - & - & - & - & 0,297 \\
\hline Cordia americana $\mathrm{L}$. & 0,087 & - & 0,185 & - & - & - & 0,272 \\
\hline Myrocarpus frondosus Allemao & 0,047 & 0,170 & - & - & - & - & 0,217 \\
\hline Jacaranda micrantha Cham. & 0,172 & 0,039 & - & - & - & - & 0,211 \\
\hline Annona cf. salicifolia Schtdl. & - & 0,175 & - & - & - & - & 0,175 \\
\hline Eugenia uniflora L. & - & 0,174 & - & - & - & - & 0,174 \\
\hline Allophylus edulis (A. St.-Hill., Camb. \& A. Juss.) Radlk. & 0,148 & - & - & - & - & - & 0,148 \\
\hline Cordia trichotmoma (Vell.) Arráb. ex steud. & - & 0,144 & - & - & - & - & 0,144 \\
\hline Casearia sylvestris Sw. & 0,114 & - & - & - & - & - & 0,114 \\
\hline Total & 3,159 & 7,811 & 10,655 & 6,326 & 5,243 & 0,538 & 33,732 \\
\hline
\end{tabular}


TABELA 3: Estoque balanceado por classe de diâmetro por hectare.

TABLE 3: Stocks balanced by diameter class per hectare.

\begin{tabular}{|c|c|c|c|c|c|c|c|c|c|}
\hline \multirow{2}{*}{$\begin{array}{c}\text { Classe } \\
\text { DAP }(\mathrm{cm})\end{array}$} & \multicolumn{3}{|c|}{ Povoamento Real } & \multicolumn{3}{|c|}{ Remanescente } & \multicolumn{3}{|c|}{ Corte } \\
\hline & $\mathrm{N}$ & $\begin{array}{c}G \\
\left(m^{2} / h a\right)\end{array}$ & $\begin{array}{c}\text { Vol } \\
\left(m^{3} / \mathrm{ha}\right)\end{array}$ & $\mathrm{N}$ & $\begin{array}{c}\mathrm{G} \\
\left(\mathrm{m}^{2} / \mathrm{ha}\right)\end{array}$ & $\begin{array}{c}\text { Vol } \\
\left(\mathrm{m}^{3} / \mathrm{ha}\right)\end{array}$ & $\mathrm{N}$ & $\begin{array}{c}G \\
\left(m^{2} / h a\right)\end{array}$ & $\begin{array}{c}\text { Vol } \\
\left(\mathrm{m}^{3} / \mathrm{ha}\right)\end{array}$ \\
\hline 20 & 24 & 0,915 & 3,159 & 14 & 0,523 & 1,807 & 10 & 0,391 & 1,351 \\
\hline 30 & 28 & 1,996 & 7,811 & 10 & 0,709 & 2,774 & 18 & 1,287 & 5,037 \\
\hline 40 & 17 & 2,577 & 10,655 & 7 & 1,049 & 4,338 & 10 & 1,528 & 6,317 \\
\hline 50 & 5 & 1,477 & 6,326 & 5 & 1,361 & 5,826 & 0 & 0,117 & 0,5 \\
\hline 60 & 3 & 1,243 & 5,243 & 4 & 1,401 & 5,912 & 0 & $-0,159$ & $-0,67$ \\
\hline 70 & 1 & 0,205 & 0,538 & - & - & - & - & - & - \\
\hline Total & 78 & 8,412 & 33,732 & 39 & 5,043 & 20,658 & 39 & 3,164 & 12,536 \\
\hline
\end{tabular}

Em que: $\mathrm{N}$ = número de indivíduos; $\mathrm{G}$ = área basal; Vol = volume.

TABELA 4: Volume por espécie classificadas para o corte por hectare.

TABLE 4: Volume by species classified to cut per hectare.

\begin{tabular}{lcccccc}
\hline \multirow{2}{*}{ Espécie } & \multicolumn{5}{c}{ Centro Classe DAP (cm) } & \multicolumn{2}{c}{$\begin{array}{c}\text { Total Geral } \\
\left(\mathrm{m}^{3} / \mathrm{ha}\right)\end{array}$} \\
\cline { 2 - 8 } & 20 & 30 & 40 & 50 & 70 \\
\hline Enterolobium contortisiliquum (Vell.) Morong. & - & 0,9468 & 5,1876 & 1,0809 & - & 7,2153 \\
Ocotea puberula (Rich.) Nees & 0,7099 & 1,8954 & - & - & - & 2,6053 \\
Desconhecida & 0,3767 & 0,4760 & - & - & - & 0,8528 \\
Parapiptadenia rigida (Benth.) Brenan & 0,0708 & - & - & - & 0,5377 & 0,6085 \\
Nectandra megapotamica (Spreng.) Mez & - & 0,0563 & 0,3181 & - & - & 0,3744 \\
Cupania vernalis Cambess. & - & 0,2053 & - & - & - & 0,2053 \\
Machaerium stipitatum (DC) Vogel & - & 0,1862 & - & - & - & 0,1862 \\
Matayba eleagnoides Radlk. & - & 0,1585 & - & - & - & 0,1585 \\
Aiouea saligna Meisn. & - & 0,1257 & - & - & - & 0,1257 \\
Allophylus edulis (A. St.-Hill., Camb. \& A. Juss.) & 0,0885 & - & - & - & - & 0,0885 \\
Radlk. & 0,0605 & - & - & - & - & 0,0605 \\
Casearia sylvestris Sw. & 0,0288 & - & - & - & - & 0,0288 \\
Jacaranda micrantha Cham. & 1,3353 & 4,0502 & 5,5057 & 1,0809 & 0,5377 & 12,5098 \\
Total Geral & & & & & & \\
\hline
\end{tabular}

tes em formato shapefile. Os rios e estradas foram definidos pelo caminhamento com o receptor GPS. As APPs foram criadas mediante a aplicação de Buffers de 15 metros, em cada lado, a partir da calha do rio (Rio $<10 \mathrm{~m}$ de largura, APP $=30 \mathrm{~m}$, Lei 4.771/65). As benfeitorias foram demarcadas com pontos de GPS, enquanto os açudes e os limites da propriedade foram gerados pela edição de polígonos criados a partir de pontos extremos destes PIs. Todos os PIs foram gerados com Datum horizontal SIRGAS 2000, no sistema de Projeção Cartográfica Transversa de Mercator, fuso 22J.

\section{Clareiras de liberação e de impacto}

As clareiras de liberação foram definidas como as áreas projetadas pelas copas das árvores selecionadas para o corte. Assim, as clareiras de liberação foram proporcionais aos diâmetros de copa calculados para cada árvore pela fórmula descrita por Longhi (1980), que correlaciona o DAP com o diâmetro de copa (Dc) pela Equação 2:

$$
D c=-1,59222+0,35060 \times D A P-0,00187 \times\left(D A P^{2}\right)
$$


Esta fórmula serviu para simular a área de clareira formada pela ausência da respectiva copa no dossel. O processamento consistiu na geração de buffers circulares, em cada ponto de árvore, no programa ArcGIS 9.2, extensão ArcToolbox >> Analysis tools $>>$ Proximity $>>$ Buffer.

As clareiras de impacto foram definidas como as áreas das secções transversais das árvores, decorrentes da queda, projetadas no solo. $\mathrm{O}$ abate dirigido foi o critério escolhido para direcionar a queda das árvores exploráveis, levando em consideração a declividade do terreno e os locais selecionados, orientados a reduzir o impacto sobre as remanescentes, facilitar o arraste e reduzir o tamanho das clareiras (SABOGAL et al., 2000).

O mapa de declividade foi gerado por meio da vetorização das curvas de nível da carta topográfica Santa Maria MI - 2665/1, escala 1:50.000, e posterior elaboração do modelo digital do terreno (MDT) pelo método da rede triangular irregular (TIN).

As áreas das secções transversais das árvores, usadas para a simulação das clareiras de impacto, foram compostas de duas partes: as secções transversais dos fustes e as secções transversais das copas. As secções transversais dos fustes foram geradas pela criação de buffers lineares, correspondentes aos diâmetros das árvores, enquanto as secções transversais das copas foram geradas por buffers circulares, a partir dos pontos localizados na metade da altura das copas, correspondentes aos diâmetros de copa, calculados pela Equação 2. A união destes buffers corresponde às secções transversais das árvores, isto é, às áreas de clareiras de impacto. $\mathrm{O}$ cálculo das áreas de clareiras de liberação e impacto formadas foi processado por intermédio da extensão Xtools Pro 4.0.

A partir das clareiras simuladas pela exploração foi possível processar as contagens dos números de indivíduos e dos valores de volume, por espécies, beneficiados pelas clareiras de liberação, bem como, daquelas atingidas pela área projetada das árvores selecionadas para o corte. O processamento foi realizado na ferramenta ArcToolbox $>>$ Analysis tools $>>$ Overlay $>>$ Spatial Join. Esta ferramenta une a tabela de atributos de diferentes feições, criando um campo, com base na localização relativa das feições dentro de dois layers, em que se atribui um valor 1 à feição (árvores) pertencente ao polígono selecionado (clareira) e 0 , caso contrário.

\section{RESULTADOS E DISCUSSÕES}

As clareiras foram classificadas em três classes de dimensão: pequenas $\left(\leq 50 \mathrm{~m}^{2}\right)$, médias $\left(51-150 \mathrm{~m}^{2}\right)$ e grandes $\left(>150 \mathrm{~m}^{2}\right)$, conforme o preconizado por Lima (2005) e Zhu et al. (2009). As clareiras de liberação foram contempladas por duas classes: clareiras pequenas (53) e médias (29). A maior frequência de clareiras de impacto foi na classe de clareiras pequenas, representada por 49 clareiras, seguida das classes de clareiras médias (32) e grandes (1) (Tabela 5). Em área, em torno de $60 \%$ da área total das clareiras formadas foram classificadas como média, tanto para as clareiras de liberação quanto para as clareiras de impacto. Segundo Tabarelli e Montovoani (1997), as clareiras pequenas e médias são mais abundantes e têm impactos menos significativos, proporcionando danos às copas, especialmente. Contudo, são as grandes que cobrem uma superfície maior e apresentam um maior distúrbio ecológico, levando à mortalidade das árvores remanescentes, devido às dimensões do impacto.

A soma das áreas de clareiras de liberação e impacto foi de $4214,21 \mathrm{~m}^{2}$ e $4318,21 \mathrm{~m}^{2}$, respectivamente. Entretanto, devido à sobreposição das áreas de copa, as áreas das clareiras foram inferiores aos valores apresentados pela soma individual das áreas de clareira e, por isso, a área de clareira de liberação simulada foi de $3382,96 \mathrm{~m}^{2}$ enquanto a área de clareira de impacto foi de $3361,39 \mathrm{~m}^{2}$.

TABELA 5: Frequências absolutas por classe de tamanho de clareiras.

TABLE 5: Absolute frequencies by size class gaps.

\begin{tabular}{cccccccccc}
\hline \multirow{2}{*}{ Classe } & \multicolumn{3}{c}{ Clareira de Liberação } & \multicolumn{5}{c}{ Clareira de Impacto } \\
\cline { 2 - 10 } & $\leq 50 \mathrm{~m}^{2}$ & $51-150 \mathrm{~m}^{2}$ & $>150 \mathrm{~m}^{2}$ & Total & Classe & $\leq 50 \mathrm{~m}^{2}$ & $51-150 \mathrm{~m}^{2}$ & $>150 \mathrm{~m}^{2}$ & Total \\
\hline $\mathrm{N}$ & 53 & 29 & - & 82 & $\mathrm{~N}$ & 49 & 32 & 1 & 82 \\
Média $\left(\mathrm{m}^{2}\right)$ & 32,41 & 86,09 & - & 59,25 & Média $\left(\mathrm{m}^{2}\right)$ & 31,86 & 81,45 & 150,54 & 87,95 \\
Desvio $\mathrm{P}\left(\mathrm{m}^{2}\right)$ & 12,39 & 26,77 & - & 13,06 & $\begin{array}{c}\text { Desvio } \mathrm{P} \\
\left(\mathrm{m}^{2}\right)\end{array}$ & 12,19 & 25,56 & - & 12,58 \\
Soma $\left(\mathrm{m}^{2}\right)$ & 1717,63 & 2496,58 & - & 4214,21 & Soma $\left(\mathrm{m}^{2}\right)$ & 1561,13 & 2606,54 & 150,54 & 4318,21 \\
\hline
\end{tabular}


As médias das áreas de clareiras de liberação e impacto foram, respectivamente, de $59,25 \mathrm{~m}^{2}$ e $87,95 \mathrm{~m}^{2}$. Estes valores são próximos aos encontrados por Lima (2005) em uma reserva de Mata Atlântica com altura de dossel variando de 20 a $40 \mathrm{~m}$, em que as áreas de clareiras variaram de 55,98 - 88,28 $\mathrm{m}^{2}$, dependendo do método empregado para gerar as estimativas. Porém, as médias foram inferiores aos $95,85 \mathrm{~m}^{2}$ encontrados por Tabarelli e Montovani (1997), em clareiras naturais, na Floresta Ombrófila Montana do Parque Estadual da Cantareira.

As árvores beneficiadas pela liberação do dossel podem ser observadas na Figura 1. A soma das áreas de clareiras de liberação foi de $3382,96 \mathrm{~m}^{2}$. Este valor está próximo à soma de abertura de dossel de 2561,1 - 3451,8 $\mathrm{m}^{2}$, de uma Floresta Temperada Montana na China, com altura de dossel variando de 15 a 20 m, encontrado por Zhu et al. (2009). No total, foram observadas 98 árvores que se encontram sob as projeções das copas das árvores a serem aba- tidas (Tabela 6). Destas, 62 indivíduos (63\%), foram classificadas com qualidade média de fuste (tortuoso e/ou bifurcado). Os indivíduos desejáveis, ou seja, com boa qualidade de fuste foram aproximadamente $30 \%$ do total. A timbaúva (Enterolobium contortisiliquum) foi a espécie que mais será beneficiada pela liberação do dossel, contemplada por 48 indivíduos, aproximadamente $50 \%$ do total, seguida da canela-guaicá (Ocotea puberula), com 13 indivíduos (13,25\%).

Em volume comercial com casca, as espécies que mais serão beneficiadas com as clareiras de liberação foram:

- Enterolobium contortisiliquum, com um volume de $14,13 \mathrm{~m}^{3}$ (mais do que dois quintos do total);

- Parapiptademia rigida, com volume de $4,39 \mathrm{~m}^{3}$;

- Phytolacca dioica, com volume de 3,89 $\mathrm{m}^{3}$;

Nota-se que as espécies beneficiadas são, em geral, de maior valor comercial, excetuando-se
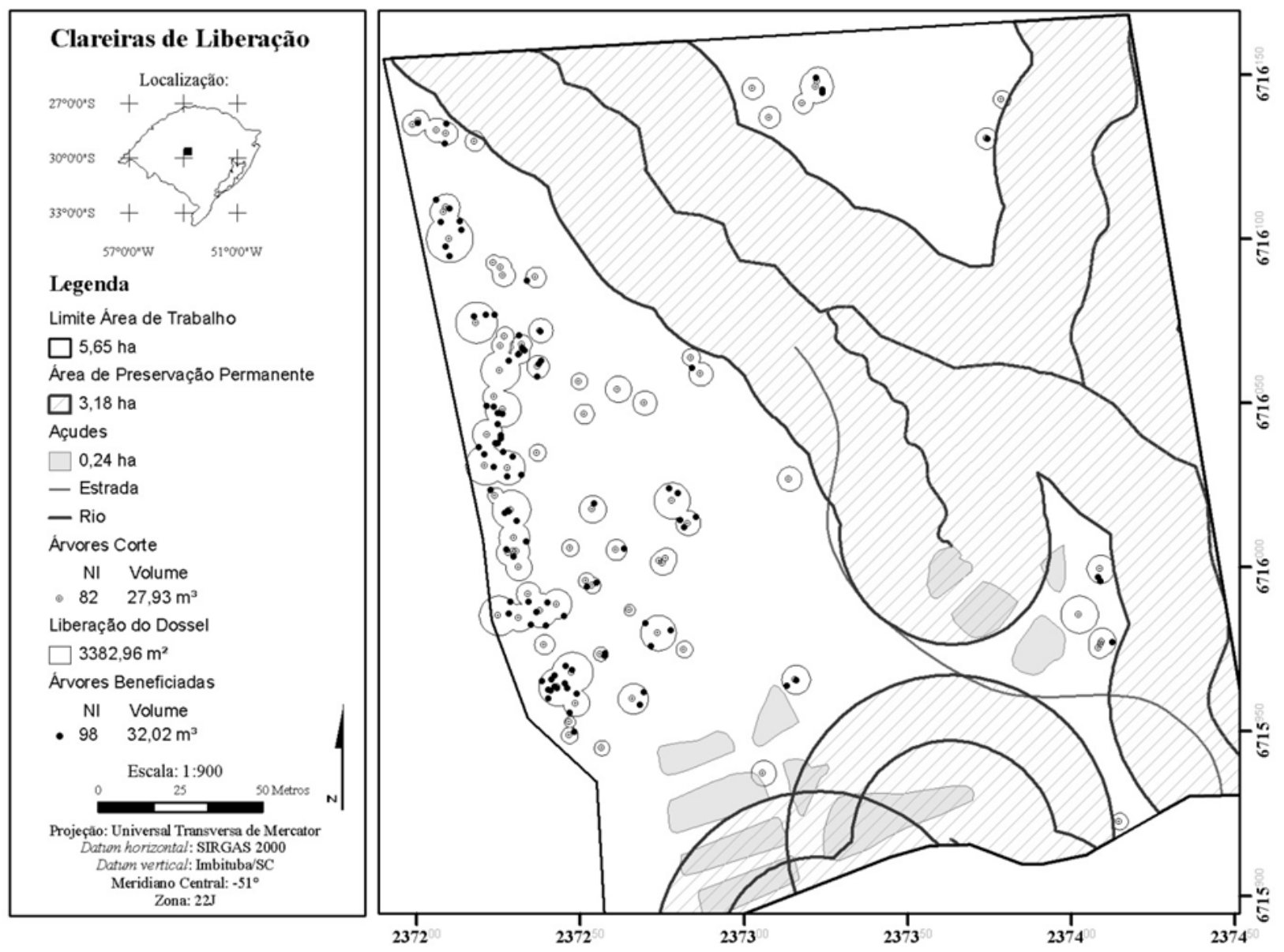

FIGURA 1: Árvores beneficiadas pela liberação do dossel pelas árvores retiradas.

FIGURE 1: Trees benefiting from the canopy release of removed trees. 
o umbu (Phytolacca dioica) que não apresenta relevância econômica. Das espécies com boa qualidade do fuste, a timbaúva contempla $70 \%$ dos indivíduos. O camboatá-branco (Matayba eleagnoides) foi representado por dois indivíduos e os demais foram contemplados por apenas um.

O diâmetro médio das árvores que serão beneficiadas pela liberação do dossel foi de $27,48 \mathrm{~cm}$ e a altura total média foi de $12,04 \mathrm{~m}$, pertencentes à faixa da classe de diâmetro $(25-34,5 \mathrm{~cm})$ - menores classes de DAP consideradas na análise - no qual o incremento corrente anual percentual em volume com casca por hectare calculado foi máximo neste trabalho $(\mathrm{ICA}=0,56 \%)$. Valor este inferior ao ICA $=1,96 \%$ da floresta estacional semidecidual no Mato Grosso (PANTALEÃO et al., 2008) e ao ICA $=1,62 \%$ da floresta tropical primária no Amazonas (TEIXEIRA et al., 2007).

$\mathrm{Na}$ Figura 4 são observadas as áreas de clareiras de impacto e a localização das árvores atingidas pela queda. Ao todo, 63 indivíduos se- rão atingidos pela queda das árvores, pertencentes a 20 espécies botânicas. Destas, aproximadamente, $75 \%$ encontram-se classificadas com média qualidade de fuste (Tabela 7).

Os indivíduos danificados correspondem a $36,7 \%$ do total dos remanescentes, sendo este valor superior aos valores de 30\% de danos registrados por Sist et al. (1998) e Vidal et al. (2002) para áreas sob planejamento e, inferiores aos $44,5 \%$ encontrados por Bulfe et al. (2009), utilizando-se de EIR, em Misiones/Argentina.

Das espécies atingidas, destaca-se a timbaúva (Enterolobium contortisiliquum) e a canela-guaicá (Ocotea puberula), com 18 e 15 indivíduos, respectivamente. Estas mesmas espécies contemplaram os maiores números de indivíduos classificados com qualidade de fuste média, 14 e 13, respectivamente.

A área basal impactada, pós-exploratória, foi de $1,57 \mathrm{~m}^{2} / \mathrm{ha}$. Este valor foi próximo ao encontrado por Bulfe et al. (2009) em uma floresta estacional semidecidual da província de Misiones,

TABELA 6: Volume por espécie e qualidade de fuste das árvores beneficiadas pela liberação do dossel.

TABLE 6: Volume by species and stem quality of the trees benefiting from the canopy release.

\begin{tabular}{|c|c|c|c|c|c|c|c|c|}
\hline \multirow{2}{*}{ Espécie } & \multicolumn{2}{|c|}{ QF (1) } & \multicolumn{2}{|c|}{ QF (2) } & \multicolumn{2}{|c|}{ QF (3) } & \multicolumn{2}{|c|}{ Total } \\
\hline & $\mathrm{N}$ & $\mathrm{V}\left(\mathrm{m}^{3}\right)$ & $\mathrm{N}$ & $\mathrm{V}\left(\mathrm{m}^{3}\right)$ & $\mathrm{N}$ & $V\left(m^{3}\right)$ & $\mathrm{N}$ & $\mathrm{V}\left(\mathrm{m}^{3}\right)$ \\
\hline Enterolobium contortisiliquum (Vell.) Morong. & 19 & 7,209 & 25 & 6,295 & 4 & 0,630 & 48 & 14,134 \\
\hline Parapiptadenia rigida (Benth.) Brenan & - & - & 3 & 4,394 & - & - & 3 & 4,394 \\
\hline Phytolacca dioica $\mathrm{L}$. & - & - & 3 & 3,891 & - & - & 3 & 3,891 \\
\hline Nectandra lanceolada Nees \& Mart. Ex Nees & 1 & 0,894 & 1 & 2,862 & - & - & 2 & 3,756 \\
\hline Ocotea puberula(Rich.) Nees & - & - & 12 & 1,709 & 1 & 0,048 & 13 & 1,756 \\
\hline Nectandra megapotamica (Spreng.) Mez & 1 & 0,814 & 1 & 0,137 & - & - & 2 & 0,951 \\
\hline Desconhecida & 1 & 0,108 & 6 & 0,662 & - & - & 7 & 0,770 \\
\hline Morta & - & - & - & - & 4 & 0,566 & 4 & 0,566 \\
\hline Matayba eleagnoides Radlk. & 2 & 0,315 & 1 & 0,066 & - & - & 3 & 0,381 \\
\hline Apuleia leiocarpa (Vogel) J. F. Macbr. & - & - & 3 & 0,337 & - & - & 3 & 0,337 \\
\hline Cordia trichotmoma (Vell.) Arráb. ex steud. & 1 & 0,213 & - & - & - & - & 1 & 0,213 \\
\hline Eribotrya japonica Lind. & - & - & 1 & 0,170 & - & - & 1 & 0,170 \\
\hline Aiouea saligna Meisn. & 1 & 0,104 & - & - & - & - & 1 & 0,104 \\
\hline Helietta apiculata Benth. & - & - & 1 & 0,090 & - & - & 1 & 0,090 \\
\hline Casearia sylvestris $\mathrm{Sw}$. & - & - & 1 & 0,064 & - & - & 1 & 0,064 \\
\hline Cordia americana $\mathrm{L}$. & - & - & 1 & 0,061 & - & - & 1 & 0,061 \\
\hline Sebastiania commersoniana (Baill.) L. B. Smith \& Downs & - & - & 1 & 0,058 & - & - & 1 & 0,058 \\
\hline Campomanesia xanthocarpa (Mart.) Berg. & - & - & 1 & 0,053 & - & - & 1 & 0,053 \\
\hline Jacaranda micrantha Cham. & - & - & 1 & 0,049 & - & - & 1 & 0,049 \\
\hline Total & 27 & 9,876 & 62 & 20,896 & 9 & 1,244 & 98 & 32,016 \\
\hline
\end{tabular}

Em que: QF (1) Qualidade de fuste alta (cilíndrico, reto); QF (2) Qualidade de fuste média (tortuoso e/ou bifurcado); QF (3) Qualidade de fuste baixa (oco, podre, morto). 


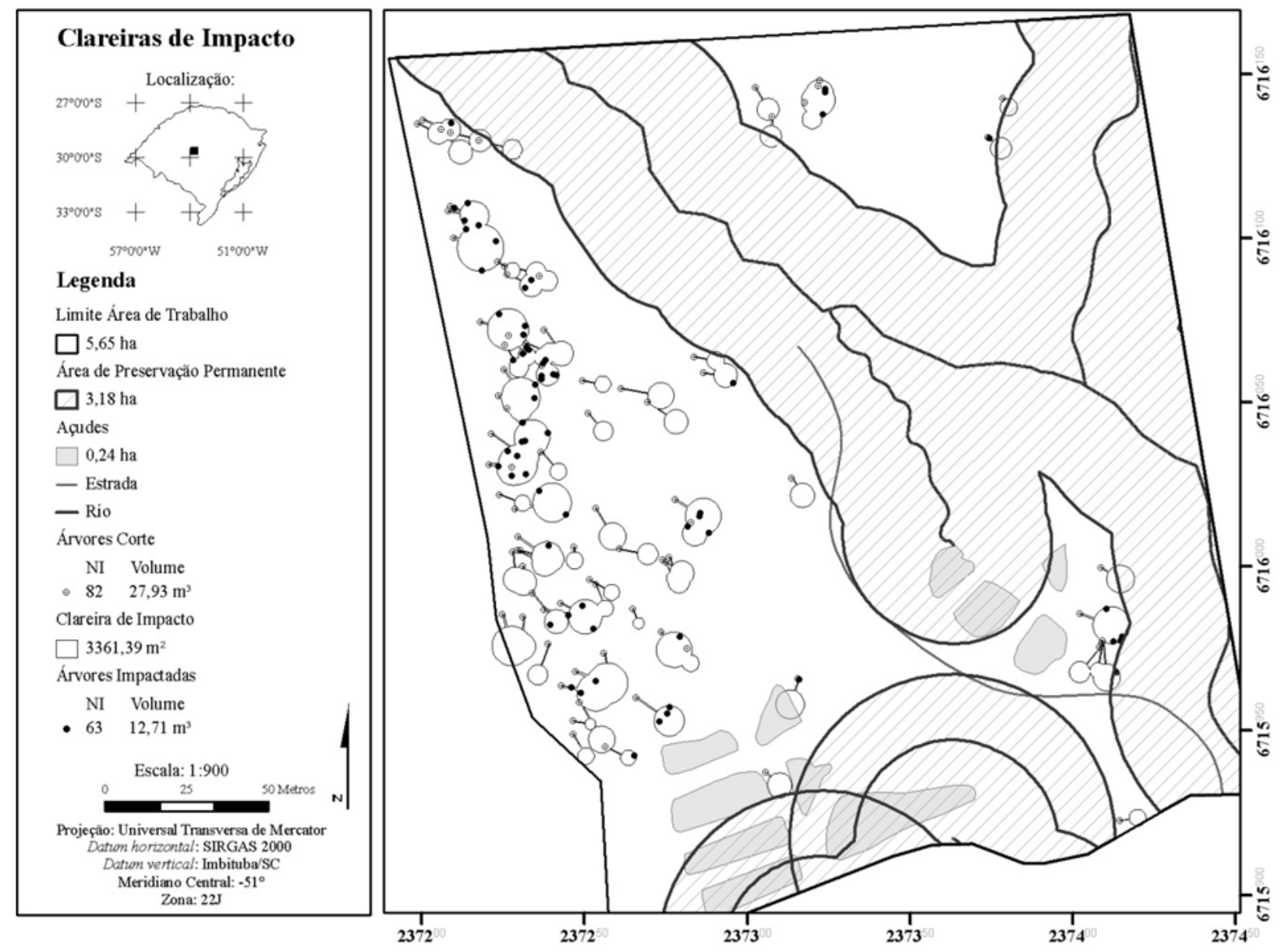

FIGURA 4: Árvores atingidas pela queda das árvores selecionadas para corte.

FIGURE 4: Trees struck by falling trees selected for cutting.

de $1,55 \mathrm{~m}^{2} /$ ha, comparando a exploração convencional e a EIR. Estes mesmos autores encontraram 13,6 ind.ha $^{-1}$ danificados pela exploração, inferior à metade do encontrado no presente trabalho (28,63 ind.ha-1).

Em volume comercial individual por espécie, a timbaúva (Enterolobium contortisiliquum) foi a espécie mais representativa, $1 / 4$ do total, seguida do umbu (Phytolacca dioica) com 1/5 do volume total e da canela-guaicá (Ocotea puberula), 1/7 do total. Estas três espécies representaram aproximadamente $65 \%$ do volume total impactado.

A área de clareira total, somando-se as áreas de clareira de liberação e impacto (excetuando-se sua sobreposição), foi de $5776,76 \mathrm{~m}^{2}$ ou $10 \%$ da área total, valor este superior ao $6,7 \%$ encontrado por Bulfe et al. (2009). Entretanto, esta discrepância pode ser justificada, pois, no estudo supracitado, somente as clareiras de impacto foram contabilizadas, excluindo-se as clareiras decorrentes da liberação do dossel. Em comparação, efetuando-se o mesmo procedimento no presente estudo, o valor seria de apenas $5,9 \%$ da área total, aproximando-se dos valores encontrados em Bulfe et al. (2009).

A média geral de diâmetro, de $23,23 \mathrm{~cm}$, observado para as árvores atingidas foi semelhante ao observado por Bertault e Sist (1997), em que as classes intermediárias de diâmetro $(30-50 \mathrm{~cm}$ de DAP) foram aquelas mais suscetíveis a danos ou injúrias. Os danos apresentados não estão de acordo com a frequência de indivíduos por classe diamétrica, pois se esperava que as classes menores (10$20 \mathrm{~cm}$ de DAP) sofressem os maiores danos por conta da maior quantidade de indivíduos nestas classes. Normalmente, as injúrias são maiores nos indivíduos de maiores dimensões, enquanto a mortalidade é mais presente nos indivíduos de menores dimensões. Árvores menores são facilmente quebradas pela queda das árvores exploradas, matando-as, ao passo que árvores maiores sofrem apenas desrama ou injúrias no tronco. Estes autores testemunharam que apenas $10 \%$ da mortalidade das árvores estão 
TABELA 7: Volume por espécie e qualidade de fuste das árvores atingidas pela queda.

TABLE 7: Volume by species and stem quality of the trees affected by the falling.

\begin{tabular}{|c|c|c|c|c|c|c|c|c|}
\hline \multirow{2}{*}{ Espécie } & \multicolumn{2}{|c|}{ QF (1) } & \multicolumn{2}{|c|}{$\mathrm{QF}(2)$} & \multicolumn{2}{|c|}{ QF (3) } & \multicolumn{2}{|c|}{ Total } \\
\hline & $\mathrm{N}$ & $\mathrm{V}\left(\mathrm{m}^{3}\right)$ & $\mathrm{N}$ & $V\left(m^{3}\right)$ & $\mathrm{N}$ & $\mathrm{V}\left(\mathrm{m}^{3}\right)$ & $\mathrm{N}$ & $V\left(m^{3}\right)$ \\
\hline Enterolobium contortisiliquum (Vell.) Morong. & 4 & 0,994 & 14 & 3,028 & - & - & 18 & 4,022 \\
\hline Phytolacca dioica $\mathrm{L}$. & - & - & 3 & 2,446 & - & - & 3 & 2,446 \\
\hline Ocotea puberula(Rich.) Nees & 2 & 0,244 & 13 & 1,483 & - & - & 15 & 1,727 \\
\hline Nectandra lanceolada Nees \& Mart. Ex Nees & 1 & 0,894 & - & - & - & - & 1 & 0,894 \\
\hline Desconhecida & 1 & 0,499 & 2 & 0,185 & - & - & 3 & 0,684 \\
\hline Hovenia dulcis Thunb. & 1 & 0,426 & - & - & - & - & 1 & 0,426 \\
\hline Cordia americana $\mathrm{L}$. & - & - & 1 & 0,407 & - & - & 1 & 0,407 \\
\hline Matayba eleagnoides Radlk. & 2 & 0,307 & 2 & 0,095 & - & - & 4 & 0,403 \\
\hline Ficus sp. & - & - & 2 & 0,403 & - & - & 2 & 0,403 \\
\hline Casearia sylvestris $\mathrm{Sw}$. & 1 & 0,073 & 2 & 0,165 & - & - & 3 & 0,238 \\
\hline Cordia trichotmoma (Vell.) Arráb. ex steud. & 1 & 0,213 & - & - & - & - & 1 & 0,213 \\
\hline Albizia niopoides (Spruce ex Benth.) Burkart & - & - & 1 & 0,125 & - & - & 1 & 0,125 \\
\hline Cupania vernalis Cambess. & - & - & 2 & 0,109 & - & - & 2 & 0,109 \\
\hline Myrocarpus frondosus Allemao & - & - & 1 & 0,108 & - & - & 1 & 0,108 \\
\hline Aiouea saligna Meisn. & 1 & 0,104 & - & - & - & - & 1 & 0,104 \\
\hline Helietta apiculata Benth. & - & - & 1 & 0,09 & - & - & 1 & 0,09 \\
\hline Morta & - & - & - & - & 1 & 0,07 & 1 & 0,073 \\
\hline Luehea divaricata Mart. & - & - & 1 & 0,067 & - & - & 1 & 0,067 \\
\hline Machaerium stipitatum (DC) Vogel & 1 & 0,062 & - & - & - & - & 1 & 0,062 \\
\hline Psidium guajava L. & - & - & 1 & 0,058 & - & - & 1 & 0,058 \\
\hline Nectandra megapotamica (Spreng.) Mez & - & - & 1 & 0,052 & - & - & 1 & 0,052 \\
\hline Total & 15 & 3,817 & 47 & 8,821 & 1 & 0,07 & 63 & 12,71 \\
\hline
\end{tabular}

Em que: QF (1) Qualidade de fuste alta (cilíndrico, reto); QF (2) Qualidade de fuste média (tortuoso e/ou bifurcado); QF (3) Qualidade de fuste baixa (oco, podre, morto).

relacionadas à queda das árvores e os restantes $90 \%$ ocorrem na etapa de arraste (skidding). Segundo os mesmos autores, dessa forma, resulta essencial o monitoramento e os tratamentos silviculturais das árvores futuro, uma vez que permitiriam melhorar as técnicas EIR, ajudando na redução dos danos e na intensidade ótima de exploração visando à sobrevivência e ao crescimento dos indivíduos remanescentes para o próximo ciclo.

\section{CONCLUSÕES}

O uso de tecnologias convergentes SIG, GPS e de Geoprocessamento para a simulação das clareiras formadas pós-exploração se mostrou satisfatório, pois a área de clareira de impacto simulada foi inferior à área da clareira de liberação (3361,39 $\mathrm{m}^{2}$ contra $\left.3382,96 \mathrm{~m}^{2}\right)$, justificando o uso do abate dirigido como técnica de EIR para reduzir o dano às árvores remanescentes. Além disso, o número de árvores atingidas pela queda foi bem inferior ao número de árvores beneficiadas pela liberação do dossel (63 contra 98), inferindo que os danos às árvores remanescentes serão menores do que os benefícios proporcionados pela liberação do dossel para árvores futuro de um eventual segundo ciclo de exploração.

O planejamento da exploração florestal, utilizando-se destas técnicas de exploração digital, permite que o analista tenha maior perspicácia nas intervenções silviculturais, não somente reduzindo os danos às árvores remanescentes, como viabilizando a perpetuação da biodiversidade, com possibilidade de espacialização de seus espécimes mais vulneráveis ou em risco. Evita-se, assim, que os cortes sejam realizados em árvores localizadas próximas à encosta de morros ou em nichos de alta biodiversidade (hot spots), por exemplo.

Recomenda-se proceder à coleta das dimensões das clareiras reais, obtidas após o abate das árvores, de forma a aproximar a situação real com as áreas e os danos simulados pela metodologia 
utilizada. Também serviria com o objetivo de minimizar os erros inerentes à precisão do GPS para o mapeamento dos indivíduos abatidos e impactados. Além disso, seria interessante realizar um inventário do banco de plântulas e/ou da regeneração natural, com a finalidade de avaliar o grau de relação entre a intensidade das intervenções silviculturais e a perpetuação da diversidade florística em função das dimensões de clareiras formadas.

\section{REFERÊNCIAS BIBLIOGRÁFICAS}

BETTS, H. D. et al. Forest canopy gap detection and characterisation by the use of highresolution Digital Elevation Models. New Zealand Journal of Ecology. v. 29, n. 1, p. 95-103. 2005.

BERTAULT, J. G.; SIST P. An experimental comparison of different harvesting intensities with reducedimpactand conventional logging in East Kalimantan, Indonesia. Forest Ecology and Management. Amsterdam, v. 94, p. 209-218, 1997. BULFE, N.M.L.Efeitos da exploração convencional e de impacto reduzido em uma floresta estacional semidecidual, na província de Misiones, Nordeste da Argentina. Floresta, Curitiba, v. 39, n. 2, p. 365379, abr./jun. 2009.

CARVALHO, J. O. P. de. Dinâmica de florestas naturais e sua implicação para o manejo florestal. In: CURSO DE MANEJO FLORESTAL SUSTENTÁVEL, 1997, Curitiba. Tópicos em manejo florestal sustentável. Colombo: EMBRAPA-CNPF, 1997. p. 43-55. (EMBRAPACNPF. Documentos, 34).

CHANDRASHEKARA,

M., RAMAKRISHNAN, P. S. Vegetation and gap dynamics of a tropical wet evergreen forest in the western ghats of Kerala, India. Journal of Tropical Ecology, v. 10, n. 3, p. 337-354, 1994.

DENSLOW, J. S. Gap partitioning among tropical rainforest trees. Biotropica, v. 12. n. 2, p. 47-55, 1980.

DURLO, M. A. Zuwachsuntersuchungen und Einzelbaumwachstumsmodelle für Cabralea glaberrima, Cedrela fissilis und Cordia trichotoma in sekundären Laubmischwälder Südbrasiliens. 1996.175 f. Dissertation (Doktorgrades) - Universität für Bodenkultur, Wien, 1996.

DURLO, M. A. Variáveis morfométricas e relações interdimensinais para Cordia trichotoma Vell. Arráb. Ex Steud. Ciência Florestal, Santa Maria, v. 19, n. 4, p 473-480, out./dez. 2009.
FIGUEIREDO, E. O. et al. Manejo de Precisão em Florestas Tropicais: Modelo Digital de Exploração Florestal. 2. ed. Rio Branco: Embrapa Acre, 2008. $183 \mathrm{p}$.

HU, L. L., ZHU, J. J. Improving gap light index (GLI) to quickly calculate gap coordinates. Canadian Journal of Forest Research, v. 38, n. 9, p. 2237-2247, 2008.

JACKSON, S. et al. Area disturbed and residual stand damage following logging in a Bolivian tropical forest. Forest Ecology and Management. Amsterdam, v. 166, p. 271-283, 2002.

JARDIM, F. S. C; VOLPATO, M. M. L; SOUZA, A. L. Dinâmica de sucessão natural em clareiras de florestas tropicais. Viçosa: SIF, 1993. 60 p. (Documento SIF 0,10)

LIMA, R. A. F. Gap size measurement: The proposal of a new field method. Forest Ecology and Management. Amsterdan, p 413-419, 2005.

LONGHI, S. J. A estrutura de uma floresta natural de Araucaria angustifolia (Bert.) $O$. Ktze no sul do Brasil. 1980. 198 f. Dissertação (Mestrado em Ciências Florestais) - Universidade Federal do Paraná, Curitiba, 1980.

MARTINS, S. V. Aspectos da dinâmica de clareiras em uma floresta estacional semidecidual no município de Campinas, SP. 1999. 215 f. Tese (Doutorado)- Universidade de São Paulo, São Paulo, 1999.

OLIVEIRA, M. V. N.; BRAZ, E. M. Manejo florestal em regime de rendimento sustentado, aplicado à floresta do Campo Experimental da Embrapa-CPAF/AC. Rio Branco: 1998. 45 p.

PANTALEÃO et al. Avaliação da estrutura, crescimento, mortalidade e recrutamento em uma floresta semidecidual submontana na amazônia Mato-Grossense. Amazônia: Ciência \& Desenvolvimento. v. 4 n. 7. 2008.

PEREIRA, R. et al. Forest canopy damage and recovery in reduce impact and convencional selective logging in eastern Para, Brazil. Forest Ecology and Management. Amsterdam, v. 168, p. 77-89. 2002.

RIO GRANDE DO SUL. Lei $\mathbf{N}^{0} \mathbf{9 . 5 1 9}$, de 21 de janeiro de 1992. Institui o Código Florestal do Estado do Rio Grande do Sul e de outras providências. Porto Alegre, 1992. Disponível em: <http://www. sema.rs.gov.br/sema/html/lei_9519.htm>. Acesso em: 28/07/09.

RUNKLE, J. R. Gap regeneration in some oldgrowth forests of the eastern United States. Ecology, v. 62 , n. 4, p. 1041-1051, 1981. 
RUNKLE, J. R. Guidelines and sample protocol for sampling forest gaps. USDA Forest Service Pacific Northwest Research Station General Technical Report PNW-GTR-283, Portland, OR. 1992.

SABOGAL, C. et al. Diretrizes técnicas para a exploração de impacto reduzido em operações florestais de terra firme na Amazônia Brasileira. Belém: Embrapa Amazônia Oriental, 2000 (Embrapa Amazônia Oriental. Documentos, 64).

SCHNEIDER, P. R.; FINGER, C. A. G. Manejo sustentado de florestas inequiâneas heterogêneas. Santa Maria: CEPEF, 2000. 195 p.

SCHÜTZ, J. P. Le régime du jardinage. Document autographique du cours de silviculture III. Chaire de sylviculture ETH-Zürich: Chaire de Silviculture, 1989. $55 \mathrm{p}$.

SIST, P. et al. Harvesting intensity versus sustainability in Indonesia. Forest Ecology and Management, Amsterdam, v. 108, p. 251-260, 1998.

SIST, P.; NGUYEN-THÉ, N. Logging damage and the subsequent dynamics of a dipterocarp forest in East Kalimantan (1990-1996). Forest Ecology and Management, Amsterdam, v. 165, p. 85-103, 2002. TABARELLI, M.; MONTOVANI, W. Colonização de clareiras naturais na floresta atlântica no sudeste do Brasil. Revista Brasileira de Botânica, São Paulo. v. 20. n. 1. p. 57-66. 1997.
TEIXEIRA et al. Projeção da dinâmica da floresta natural de Terra-firme, região de Manaus-AM, com o uso da cadeia de transição probabilística de Markov. Acta amazonica. v. 37. n. 3. p. 377-384. 2007.

VALVERDE, T.; SILVERTOWN, J. Canopy closure rate and Forest structure. Ecology, v. 78, n. 5, p. 1555-1562, 1997.

VEBLEN, T. T. Tree regeneration responses to gaps along a transandean gradient. Ecology, v. 70, n. 3, p. 541-543, 1989.

VIDAL, E. et al. Efeitos da exploração madeireira predatória e planejada sobre a diversidade de espécies na Amazônia Oriental. Revista Árvore, v. 22, n. 4, p. 503-520, 1998.

VIDAL, E. et al. Crescimento de floresta tropical três anos após colheita de madeira com e sem manejo florestal na Amazônia oriental. Scientia Forestalis, Piracicaba, n. 61, p. 133-143, 2002.

WHITMORE, T. C. Gaps in the forest canopy. In: TOMLINSON, P. B.; ZIMMERMANN, M. H. (Eds.) Tropical trees as living systems. New York: Cambridge University Press, 1978. p. 639-655.

WHITMORE, T. C. Canopy gaps and the two major groups of forest trees. Ecology, v. 70, n. 3, p 536538, 1989.

ZHU, J. J. et al. A new calculation method to estimate forest gap size. Chinese Journal of Ecology, v. 4, n. 3, p. 276-282, 2009. 